\title{
Comparison of Digital Terrain Models obtained with LiDAR and photogrammetry
}

\author{
Martínez-Agirre Alex ${ }^{1}$, García-Morales Víctor ${ }^{2}$, Álvarez-Mozos Jesús ${ }^{1 *}$ \\ 1) Department of Engineering, Public University of Navarre, Los Tejos, Campus Ar- \\ rosadia, 31006, Pamplona, Spain \\ 2) Department of Engineering and Territorial Systems, TRACASA, Cabárceno 6, \\ 31621, Sarriguren, Spain \\ *Corresponding author: jesus.alvarez@unavarra.es
}

\begin{abstract}
Airborne LiDAR sensors capture three-dimensional information of the Earth, useful for obtaining high accuracy Digital Terrain Models (DTM). The Spanish National Plan for Aerial Orthophotography (PNOA) is an initiative of the Spanish Geographical Institute whereby nationwide LiDAR datasets are periodically acquired and made available to the public as .las files and value added products (e.g., DTM). The objective of this study is to assess the added value of PNOA LiDAR DTMs by comparing them to DTMs obtained through classical photogrammetric techniques. With this aim, four areas of interest were selected in Navarre (north of Spain), in areas with challenging characteristics such as forests, karst landforms, agricultural terraces and ravines. A 5x5 m DTM obtained with classical photogrammetry in 2008 was compared with a LiDAR DTM of the same pixel size obtained in 2011, assuming no significant changes occurred in this time. Height differences were evaluated, as well as slope, aspect and curvature differences. Besides, a multiresolution analysis was carried out to quantify how DTM smoothing affected height variations between neighbor pixels, measured with the standard deviation on a $5 \times 5$ window. The results obtained showed that the LiDAR DTMs provided an enhanced description of topography, particularly under forests and in areas with complex topography.
\end{abstract}

Keywords: LiDAR, photogrammetry, DTMs, cartography, geomorphology.

\section{Introduction}

Digital Terrain Models (DTM) represent the topographic surface as a grid of regularly spaced pixels whose values correspond to the bare earth height (removing man-made objects and vegetation) [1]. Although some other types of representations might also 
be used (e.g., irregular point clouds or TINs), the above mentioned raster model is the most frequent and convenient format to work with [1]. DTMs are extremely valuable datasets for engineering related activities occurring at the earth surface [2], as well as for many other disciplines (e.g., geomorphology, ecology, hydrology and forest management) [3]. DTMs can be obtained through different techniques [3], but at detailed scales (1:5000 or higher) aerial photogrammetry was the standard for several decades, until Light Detection And Ranging (LiDAR) surveys came up [4]. LiDAR datasets consist of an enormous amount of points with known $\mathrm{X}, \mathrm{Y}, \mathrm{Z}$ coordinates, corresponding basically to the bare earth surface, vegetation elements and man-made objects. After a filtering process whereby vegetation and man-made objects are masked out, raster DTMs can be easily obtained at typical resolutions of $5 \mathrm{~m}$ and higher depending on the density of the LiDAR point cloud.

In many countries, LiDAR surveys are becoming the standard data source for DTMs, as they have a number of advantages compared to photogrammetry. For example, the laser pulse can penetrate through vegetation and reach the soil beneath forests. In addition, photogrammetry frequently requires the intervention of a skilled analyst to delineate contour lines or to define the lines obtained (semi-)automatically [5]. However, the cost of LiDAR surveys might still be too high for under developed countries, limiting the adoption of this technology.

The objective of this study is to quantitatively compare DTMs obtained with LiDAR and photogrammetry considering areas with particular topographic features where the added value of LiDAR might be particularly apparent.

\section{Materials}

A 1:5000 scale DTM obtained through photogrammetry was used. This DTM was created through manual restitution of contour lines, key points and other planimetric elements useful for representing the topography. Different photogrammetric flights were used to build the DTM. A first model was built in 2000. It was later updated and improved with successive flights in 2003, 2004, 2006 and 2008. All flights were carried out in summer time, typically flying at a height of $\sim 5000 \mathrm{~m}$ over ground level with a $60 \%$ overlap and obtaining ground sampling distances (GSD) of $25-50 \mathrm{~cm}$. The final product was a DTM with a $5 \times 5 \mathrm{~m}$ pixel size in UTM-30N ETRS-89 projection system and orthometric heights.

On the other hand, a LiDAR was flown over Navarra between February 2011 and May 2012 as part of the Spanish National Plan for Orthophotography (PNOA) using a Leica ALS60 sensor. The flight specifications (height, speed, FoV) were set up so as a required point density of $>0.5 \mathrm{pt} / \mathrm{m}^{2}$ and a vertical accuracy of $<20 \mathrm{~cm}$ attending to the PNOA specifications. Obtained LAS point clouds were ground filtered using TerraScan software and a DTM of $5 \times 5 \mathrm{~m}$ pixel was interpolated and published in UTM$30 \mathrm{~N}$ ETRS-89 projection system and orthometric heights. This DTM is publicly available at the Spanish National Geographic Institute (www.cnig.es). 
Four $4 \mathrm{x} 4 \mathrm{~km}$ areas of interest were selected in Navarra to reflect challenging conditions for DTM extraction, regarding relief abruptness, particular landforms or dense vegetation cover (Table 1 and Fig. 1).

Table 1. Areas of interest

\begin{tabular}{cllll}
\hline Zone & Name & Xmin-Xmax & Ymin-Ymax & Description \\
\hline 1 & Oskotz & $600000-604000$ & $4756000-4760000$ & Dense forest \\
2 & Uxue & $620000-624000$ & $4706000-4710000$ & Agricultural terraces \\
3 & Larra & $680000-684000$ & $4755000-4759000$ & Karst relief \\
4 & Rincón del Bú & $620000-624000$ & $4665000-4669000$ & Arid ravines \\
\hline
\end{tabular}

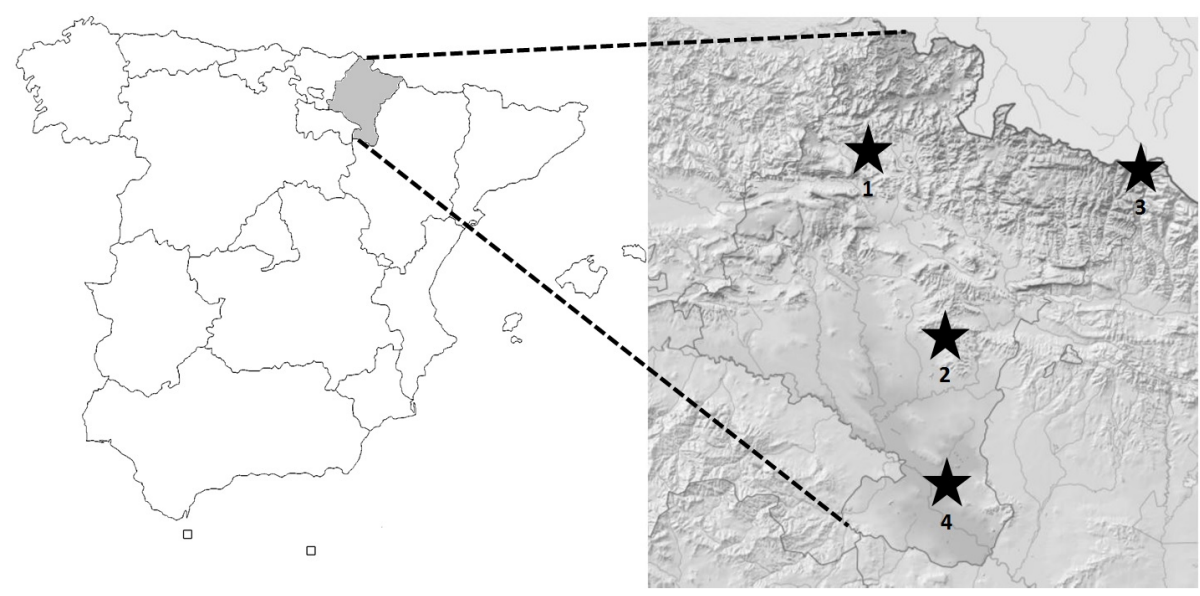

Fig. 1. Location of the four study sites in Navarre. See table 1 for more details.

\section{Methods}

The applied methodology comprised several processes (Fig. 2). DTMs were clipped and terrain features (i.e. slope, aspect and curvature) were computed following [6] for both the LiDAR and photogrammetric DTMs (from now on referred to as DTM $\mathrm{L}_{\mathrm{L}}$ and $\mathrm{DTM}_{\mathrm{P}}$, respectively). Raster differencing was applied to quantitatively evaluate DTM differences. Height differences were computed $\left(z_{\text {diff }}\right)$, as well as slope $\left(s l p_{d i f f}\right)$, aspect

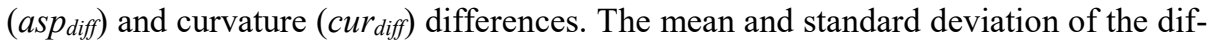
ferences were evaluated for each area of interest.

Finally, a multiresolution analysis was carried out to evaluate the level of detail of elevation data included on each DTM (Fig. 2). For this, the standard deviation of heights $\left(s_{z}\right)$ on a $5 \times 5$ window $(25 \times 25 \mathrm{~m})$ was used as a descriptor of the abruptness of 
relief or topographic roughness. Then, the original DTMs were smoothed using boxcar filters of increasing $k \times k$ window size (with $k=3,5, \ldots, 19$ ), and $s_{z}$ was successively computed for the filtered DTMs. The trend of $s_{z}(k)$ was plotted, being the slope of this trend an indicator of the elevation information lost when neighbor pixels were averaged.

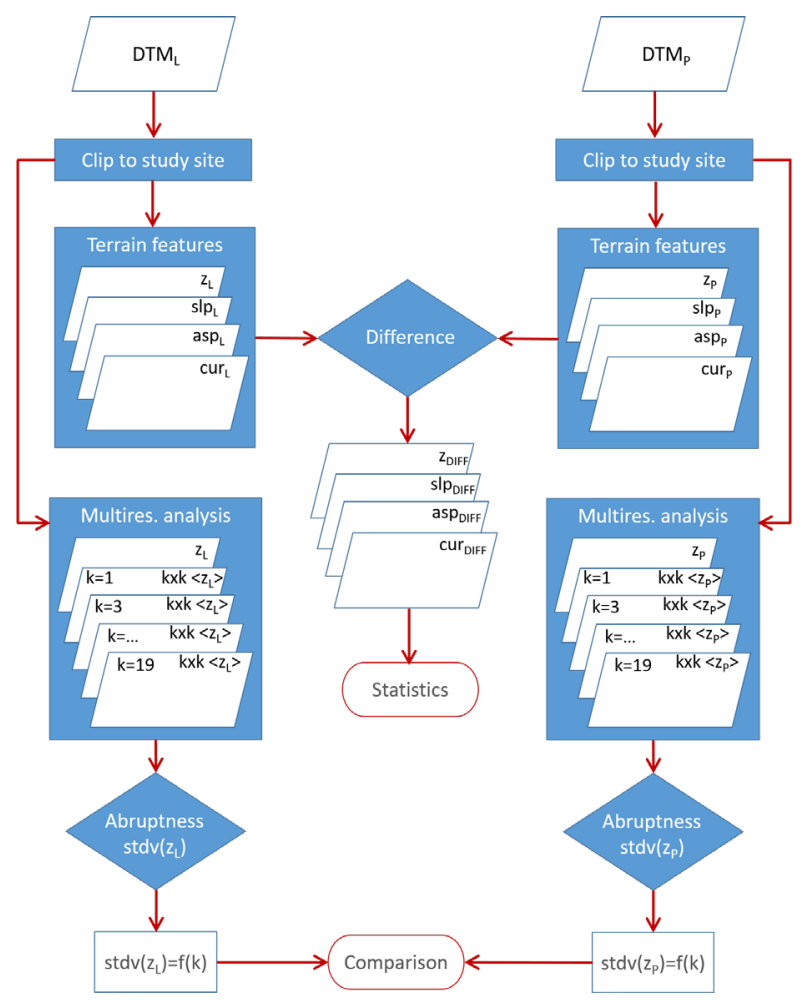

Fig. 2. Flowchart describing the methodology followed for the analysis.

\section{Results}

Figures 3-6 represent the results obtained for Zone 1, similar figures were obtained for the other three zones but only summary results were given (Table 2) for the sake of brevity. At a first sight DTMs obtained with LiDAR and photogrammetry looked quite similar (Fig. 3 top row), however a closer inspection revealed differences that were clearly depicted in $z_{\text {diff }}$ (Fig. 3 bottom row). In zone $1, z_{\text {diff }}$ took mostly negative values, corresponding to areas where $\mathrm{DTM}_{\mathrm{L}}$ was below DTMP, mostly due to the dense forest canopy in the area. 

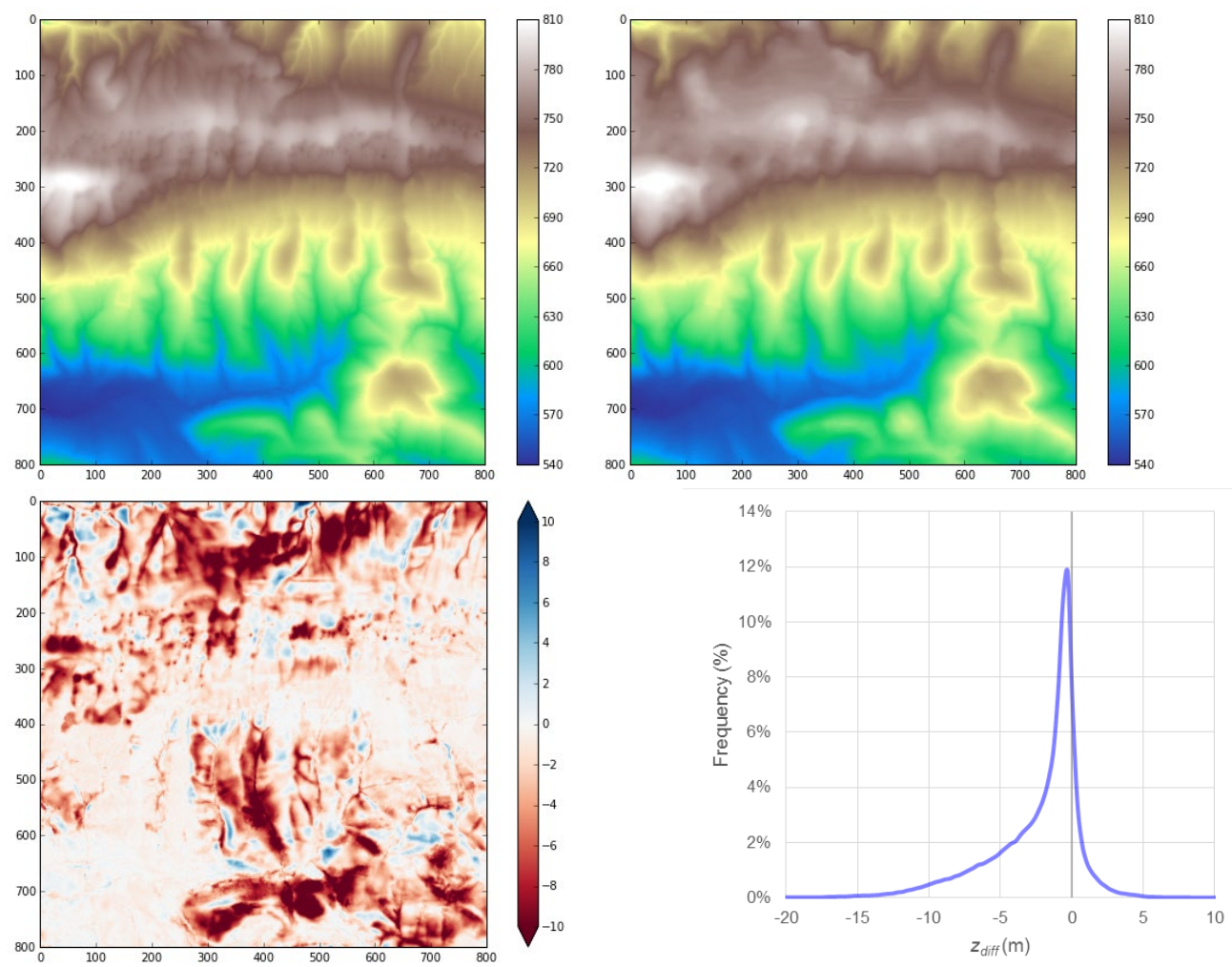

Fig. 3. DTM differences for Zone 1. Top-left: LiDAR DTM, top-right: Photo DTM, bottom-left DTM difference $\left(z_{\text {diff }}\right)$ and bottom-right $z_{\text {diff }}$ histogram.

Slope, aspect and curvature had also quite a similar appearance, but significant differences were apparent when raster differences were computed (Figs. 4-6). In the case of slope (Fig. 4), differences were centered around a mean value of $1.68 \%$ (Table 2) and positive values were more frequent, indicating, in general, steeper slopes in $\mathrm{DTM}_{\mathrm{L}}$ than in DTMP.

For the aspect (Fig. 5) the histogram of differences was rather symmetric, revealing both positive and negative aspect variations. These differences mostly corresponded to sinkholes frequent in the highlands of the mountain range and to steep valleys in the northern part of the area. As explained, this site was densely forested area and thus photogrammetry was unable to describe correctly these terrain features. The central part of zone 1, mostly occupied by grasslands, had a better agreement between photogrammetry and LiDAR. Finally, curvature differences (Fig. 6) were mostly located along linear features such as streams, forest roads and cattle tracks, where LiDAR data revealed more subtle details of topography. 

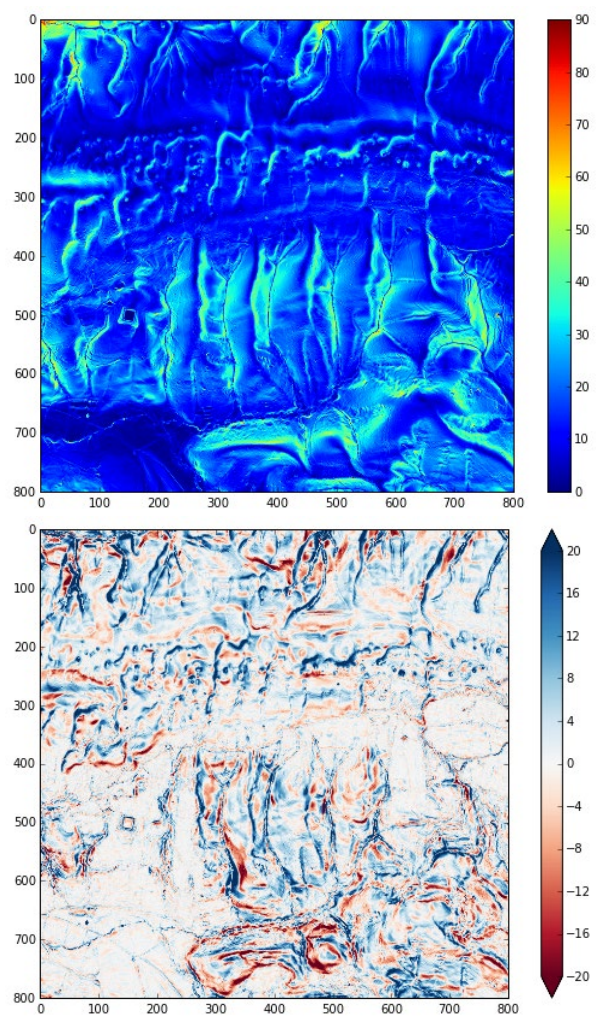
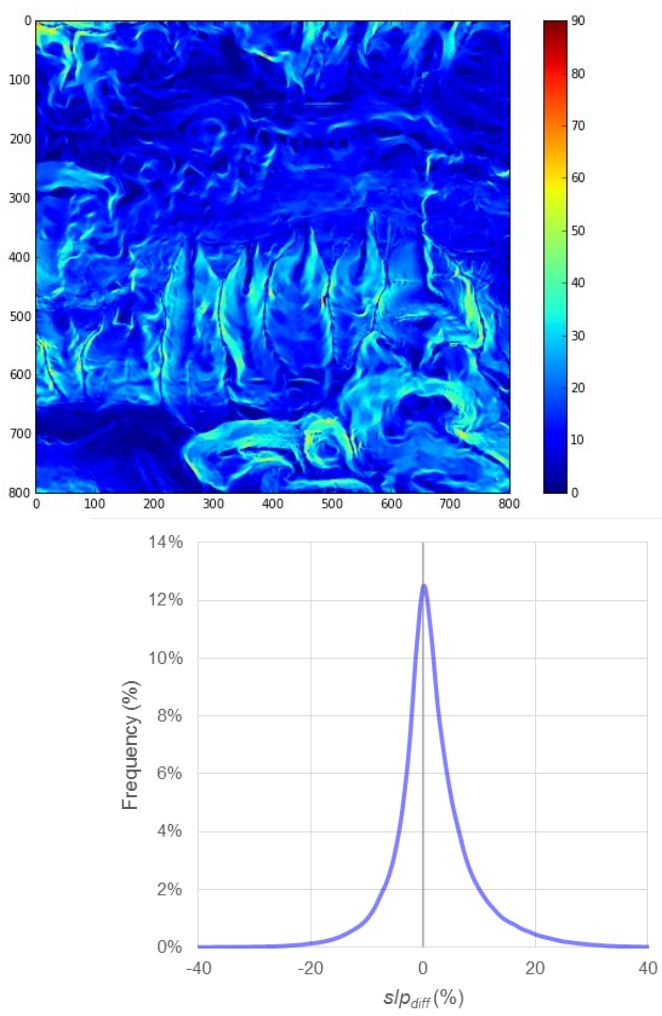

Fig. 4. Slope differences for Zone 1. Top-left: LiDAR slope, top-right: Photo slope, bottom-left slope difference $\left(s l p_{\text {diff }}\right)$ and bottom-right $s p_{\text {diff }}$ histogram.

When analyzing the results obtained for the other study zones (Table 2), it can be observed that height differences were in general smaller than for Zone 1 and with positive sign, indicating a higher $\mathrm{z}$ value for $\mathrm{DTM}_{\mathrm{L}}$. Height differences and their variations ( $s$ value in brackets in Table 2) were particularly large for Zone 3, a very abrupt karstic mountain area. In this area, slope differences were also very relevant with much steeper slope values obtained for $\mathrm{DTM}_{\mathrm{L}}$ (average value $11.30 \%$ higher). Aspect and curvature differences were also notable. Zone 2 corresponded to a terraced agricultural area, where mean differences were moderate but standard deviation values were relevant, especially for the slope, indicating that terrace limits were not adequately delineated in DTMP. Finally, Zone 4 showed the least differences between LiDAR and photogrammetry, since it corresponded to a rather flat and bare area were arid ravines exist. In this case, aspect differences were quite relevant (high standard deviation values) probably due to the difficulty of correctly computing the aspect for mostly flat areas. 

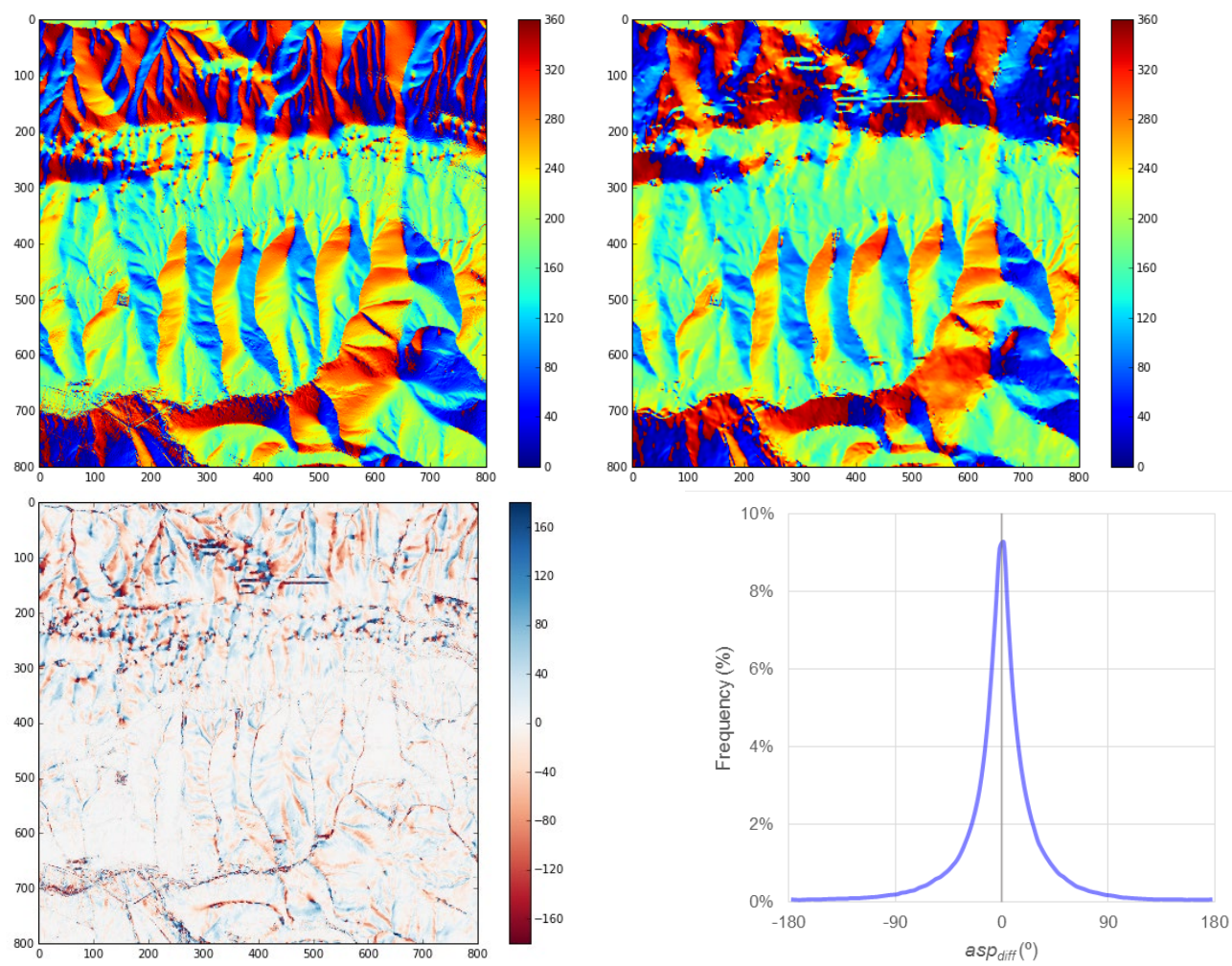

Fig. 5. Aspect differences for Zone 1. Top-left: LiDAR aspect, top-right: Photo aspect, bottom-left

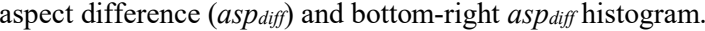

Table 2. Mean and standard deviation ( $s$ in brackets) of height, slope, aspect and curvature differences ( $z_{\text {diff }}, s l p_{\text {diff, }}, a_{s} p_{\text {diff }}$ and $c u r_{\text {diff, }}$ respectively) for the four study zones.

\begin{tabular}{cccccc}
\hline Variable & Units & Zone1 & Zone2 & Zone3 & Zone4 \\
\hline$z_{\text {diff }}$ & $(\mathrm{m})$ & $-2.37(3.20)$ & $0.92(1.38)$ & $1.63(4.04)$ & $0.45(1.08)$ \\
slp $_{\text {diff }}$ & $(\%)$ & $1.68(7.21)$ & $0.62(12.79)$ & $11.30(26.29)$ & $0.56(7.76)$ \\
asp $_{\text {diff }}$ & $\left({ }^{\circ}\right)$ & $-0.53(37.94)$ & $-0.06(26.19)$ & $0.46(49.32)$ & $-0.04(61.99)$ \\
cur $_{\text {diff }}$ & $\left(\mathrm{m}^{-1}\right)$ & $-2.5 \mathrm{E}-5(0.05)$ & $1.0 \mathrm{E}-5(0.15)$ & $3.8 \mathrm{E}-5(0.26)$ & $-6.5 \mathrm{E}-6(0.10)$ \\
\hline
\end{tabular}



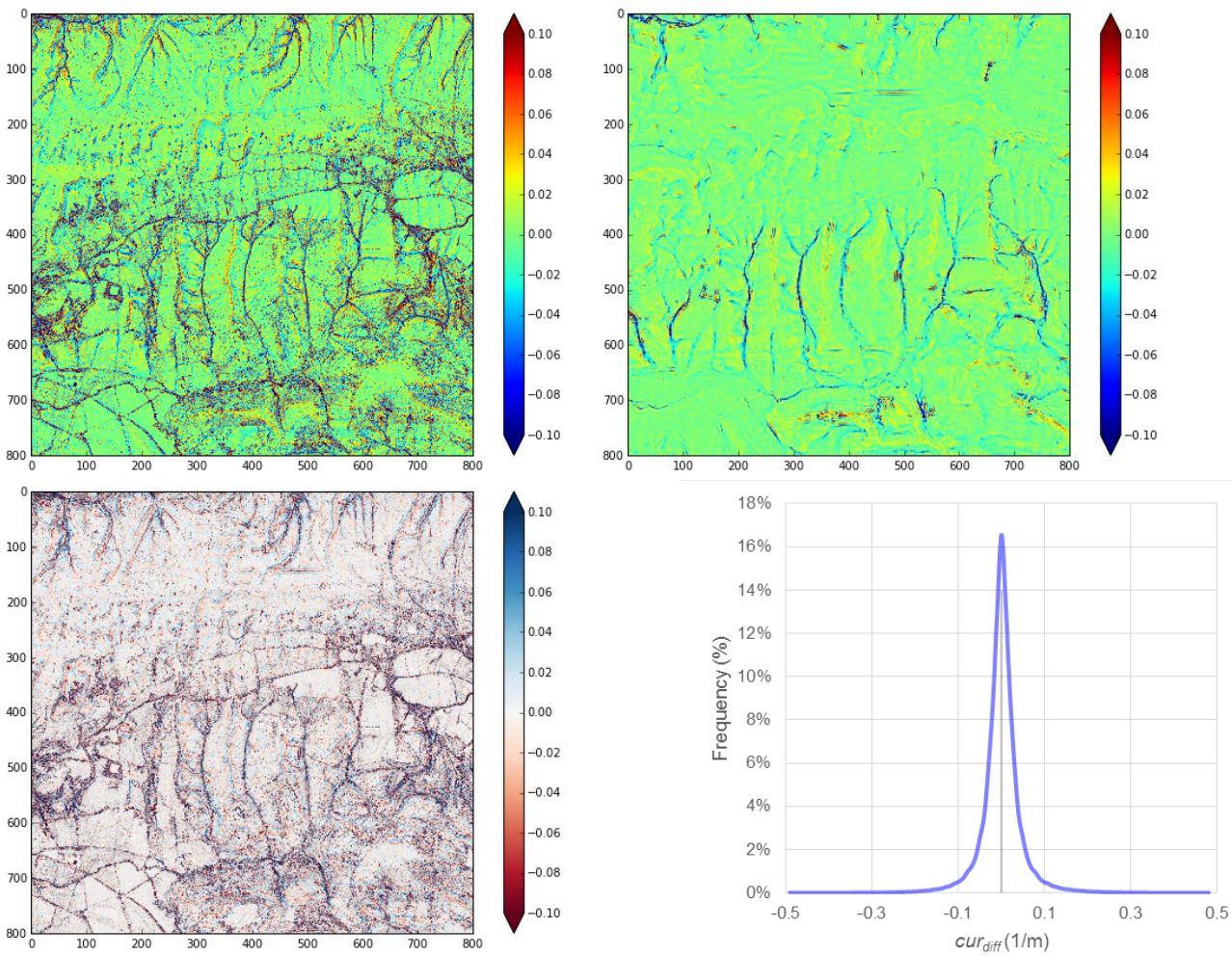

Fig. 6. Curvature differences for Zone 1. Top-left: LiDAR curvature, top-right: Photo curvature, bottom-left curvature difference ( cur $\left._{\text {diff }}\right)$ and bottom-right cur diff $_{\text {histogram. }}$.
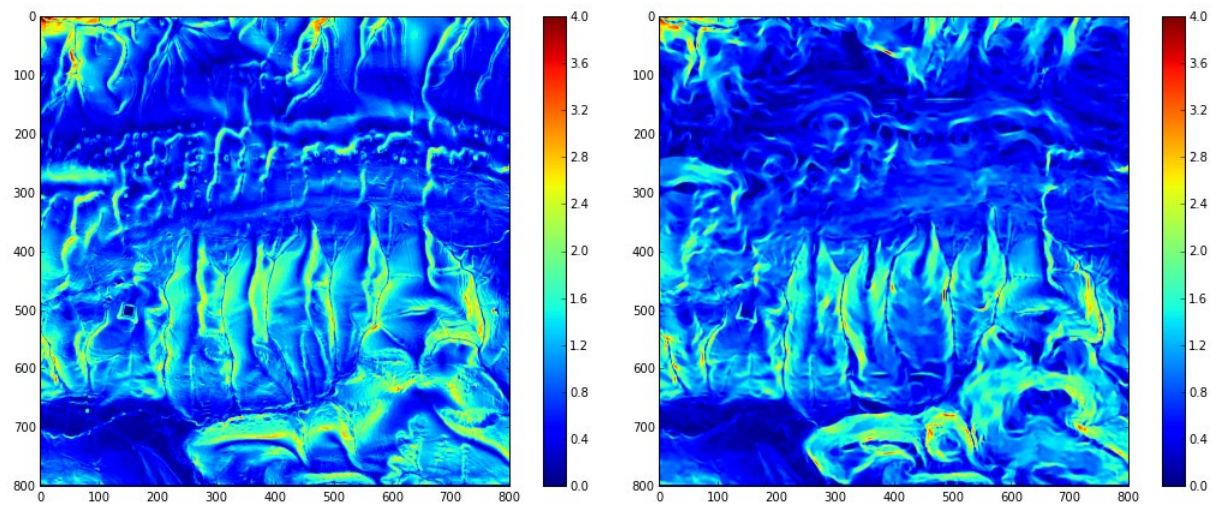

Fig. 7. Topographic roughness $\left(s_{z}\right)$ of Zone 1, as obtained with LiDAR (left) and photogrammetry (right).

Finally, the topographic roughness was evaluated by means of $s_{z}$. It can be observed that $s_{z}$ took higher values for DTM , illustrating a stronger variability of elevation 
values around neighbor pixels and thus a finer spatial detail of elevation data (Fig. 7). The difference between $s_{z}$ values obtained for DTM $\mathrm{L}_{\mathrm{L}}$ and DTM $\mathrm{P}_{\mathrm{P}}$ was particularly large for zones with stronger topography (Zone 3) and forests (Zone 1), whereas Zone 2 and particularly Zone 4 showed less differences (Fig. 8).

Fig. 8 .
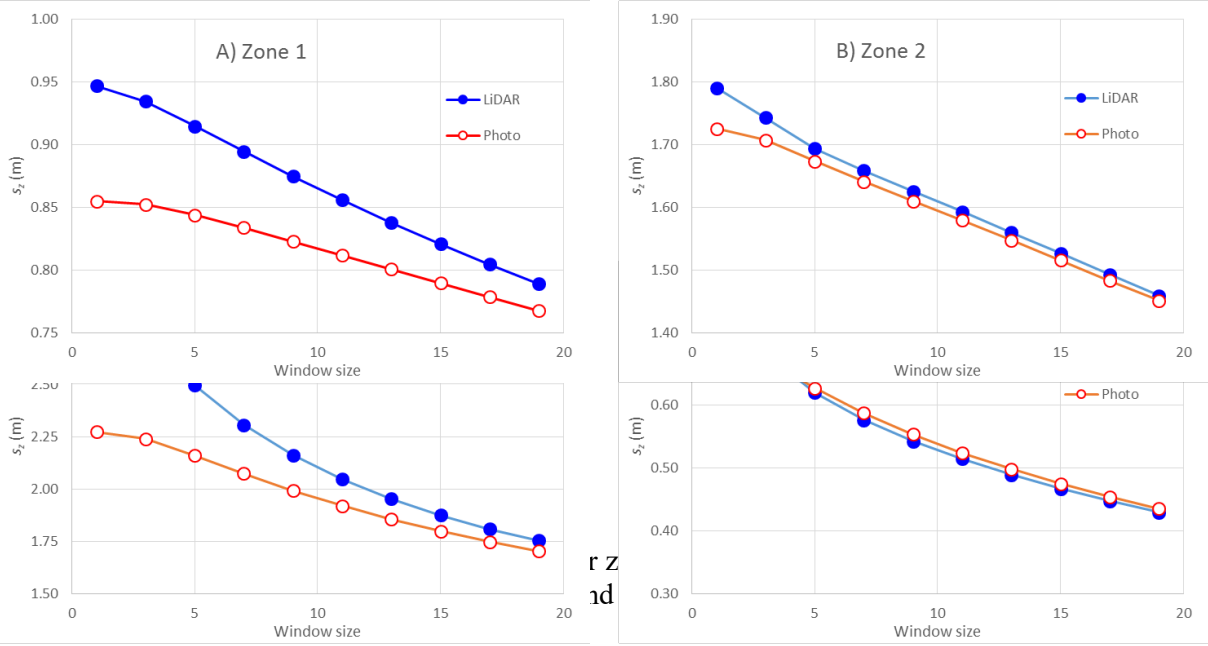

The multiresolution analysis (Fig. 8) showed a decreasing trend of $s_{z}(k)$, which was steeper for LiDAR data. This revealed a higher loss of spatial detail as DTMs were smoothed, particularly in areas with abrupt terrain (Zones 1 and 3). DTMs obtained with photogrammetry had a gentler decay of $s_{z}(k)$, especially when $k$ was small, indicating that the topographic roughness at $5 \mathrm{~m}$ resolution was mostly similar to that a resolution of $25 \mathrm{~m}(k=5)$. As $k$ values increased, LiDAR and photogrammetry trends converged, this convergence was achieved faster in Zones 2 and 4.

\section{Conclusion}

The results obtained demonstrate that LiDAR based DTMs can describe the topography at a greater detail than conventional photogrammetry, particularly in areas with strong topography and dense vegetation cover (forests). Elevation differences, as well as slope, aspect and curvature, revealed finer details in LiDAR based DTMs. These differences were confirmed when computing the topographic roughness and its decay as DTMs were smoothed with a low pass filter, showing a smoother appearance of photogrammetry based DTMs for most cases. These results recommend the use of LiDAR surveys for DTM extraction for any mapping and engineering related activity, particularly for areas with strong topography and forest cover. 


\section{Acknowledgments}

The research work reported here was partly funded by project CGL2016-75217-R (MINECO/FEDER, EU).

\section{References}

1. Li, Z., Zhu, Q., Gold, C.: Digital Terrain Modeling Principles and Methodology. 1st edn. CRC Press, Boca Raton (2004).

2. Petrie, G., Kennie, T.J.M.: Terrain modelling in surveying and civil engineering. Computer-Aided Design 19(4), 171-187 (1987).

3. Maune, F.: Digital Elevation Model Technologies and Applications: The DEM Users Manual. 3rd edn. American Society for Photogrammetry and Remote Sensing, Bethesda (2001).

4. Wack, R., Wimmer, A.: Digital terrain models from airborne laser scanner data-a grid based approach. International Archives of Photogrammetry Remote Sensing and Spatial Information Sciences XXXIV (Pt. 3B), 293-296 (2002).

5. Gil, A.L., Núñez-Casillas, L., Isenburg, M., Alonso Benito, A., Rodrigo Bello, J.J., Arbelo, M: A comparison between LiDAR and photogrammetry digital terrain models in a forest area on Tenerife Island. Canadian Journal of Remote Sensing 39(5), 396-409 (2013).

6. Zevenbergen, L.W., Thorne, C.R.: Quantitative analysis of land surface topography. Earth Surface Processes and Landforms 12, 47-56 (1987) 